\title{
Fabrication of Magnetic Force Microscopy Tips via Electrodeposition and Focused Ion Beam Milling
}

\author{
O. Céspedes, A. Luu, F. M. F. Rhen, and J. M. D. Coey \\ School of Physics, Trinity College Dublin, Dublin 2, Ireland
}

\begin{abstract}
A method is described for the fabrication of magnetic force microscopy tips via localized electrodeposition and focused ion beam milling departing from commercial tapping mode tips. Very high aspect ratios and interacting magnetic moments are possible without altering significantly the tapping resonant frequency of the cantilever. These tips can achieve high magnetic resolution at room temperature and open atmosphere. They can also be fabricated into any shape, with applications for ferromagnetic resonance measurements and nano-imprinting.
\end{abstract}

Index Terms-Electrodeposition, focused ion beam milling, magnetic force microscopy (MFM), tip fabrication.

\section{INTRODUCTION}

$\mathbf{M}$ AGNETIC force microscopy (MFM) has become the technique of choice to measure the micrometer and submicrometer magnetic configuration of thin films and smooth samples thanks to its relatively easy use and the possibility to make the measurement at room temperature and open atmosphere with little or no sample preparation. The magnetic probes are usually thin $\mathrm{Si}$ or $\mathrm{Si}_{3} \mathrm{~N}_{4}$ cantilevers finished on a pyramidal tip of atomic sharpness and covered with a magnetic thin film. These cantilevers can be made to oscillate at resonant frequencies of order $200 \mathrm{kHz}$ with spring constants of $20-50 \mathrm{~N} / \mathrm{m}$ and quality factors ranging from 1 to 100 . The most common magnetic force imaging method (Tapping-Lift Mode TM from Digital Instruments) is based on a double scanning of the sample. On the first scan, the topography of the sample is recorded by gently tapping its surface at the resonant frequency of the cantilever while keeping the amplitude of oscillation constant by moving the sample up and down with a piezoelectric element. On the following scan the instrument remembers the sample topology and it passes the cantilever at a constant height over the sample features (lift height) while maintaining a constant resonant lift-amplitude as well, so that the tip will not touch the sample but will interact via electromagnetic forces. Changes of this interaction forces can be detected via phase and frequency changes on the cantilever oscillation, which will be mostly due to the stray field gradients from the sample and can be simulated both as monopole or dipole interaction [1].

Although the resolution of this technique can routinely go down to $100 \mathrm{~nm}$ at room temperature and open atmosphere, ever-increasing data storage density and nanoscale magnetic devices demand higher sensitivity and resolution, which is hard to obtain with conventional microscopes in air. Many tip fabrication methods have been proposed to increase the aspect ratio and/or resolution of MFM of commercial MFM tips without changing its operating settings by using magnetic multilayers [2], high coercivity CoPt tips [3], magnetically filled

Digital Object Identifier 10.1109/TMAG.2008.2002866

Color versions of one or more of the figures in this paper are available online at http://ieeexplore.iee.org. carbon nanotubes [4], electrodeposited magnetic nanowires [5] and electron beam deposition [6]. A best spatial resolution of $11 \mathrm{~nm}$ has been reported for averaged scan line measurements using focused ion beam (FIB) milled CoPt tips on high density perpendicular recording media with $1000 \mathrm{kfci}$ [7] (spacing in magnetization direction about $25 \mathrm{~nm}$ ). However, all these methods limit the amount of magnetic material at the tip, which even in the case of commercial tips is below $10^{-3} \mu \mathrm{m}^{3}$. The reduced magnetic volume not only may affect the sensitivity of the tip on low moment or in-plane magnetization samples, but also may alter its hard magnetic properties, reducing its coercivity. Furthermore, new applications for magnetic and atomic force microscopy such as ferromagnetic resonance measurements [8], [9], magnetic nano-writing [10] and force lithography [11] have specifications that require or would benefit from high net magnetic volumes, high coercivity, and high aspect ratios, respectively.

To enhance the aspect ratio and sensitivity of commercial MFM tips while at the same time increasing their magnetic volume to maximize the resolution and possible applications, we have developed a fabrication method using commercial tapping mode cantilevers, localized electrodeposition and focused ion beam milling. Following this method we have successfully fabricated MFM tips of nickel and CoPt with a magnetic volume of order $100 \mu \mathrm{m}^{3}$, high aspect ratio and good sensitivity and resolution at room temperature on air.

\section{DESCRIPTION OF THE FABRICATION METHOD}

As a general rule, the sharper an MFM tip is, the better the resolution it provides. However, the main problem encountered when sharpening commercial MFM tips using focused ion beam milling is the unavoidable loss of magnetic material and signal strength. One approach is to plate a thick magnetic film and latter sharpen it using FIB. It has been demonstrated that thick electrodeposited films with high coercivity such as CoPt [12] and CoPtP [13] can be obtained, with CoPtP coercivities up to almost $0.7 \mathrm{~T}$ in the as-deposited state. Atomic force microscopy tips have also been prepared by electrodeposition before [14], but those tips can only be used in contact mode and do not have applications in magnetic microscopy. For the fabrication of high coercivity MFM tips we could deposit a thick layer of CoPtP (i.e., $1 \mu \mathrm{m}$ or more) over a tapping tip, but in our experience this method does not give good results as the thick layer alters 


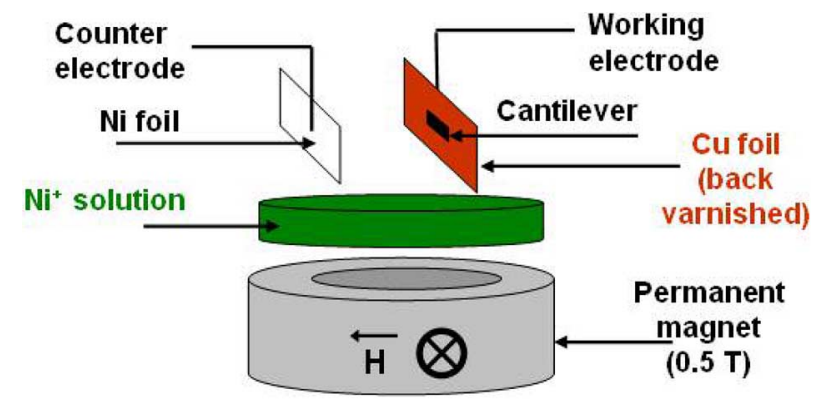

Fig. 1. Schematic for the electrodeposition of nickel on AFM cantilevers. The magnetic field is used to avoid excessive electric field gradients than can damage the cantilever and to easy the flow of nickel grains towards it.

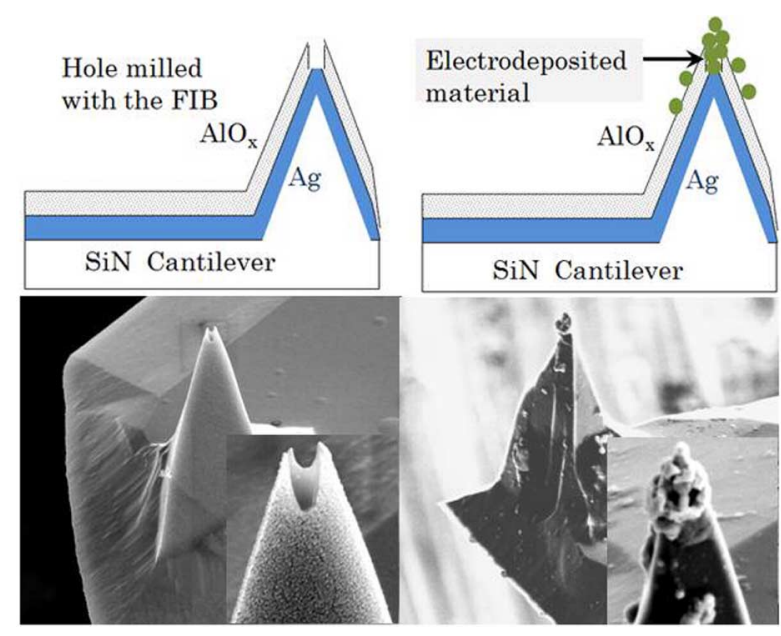

Fig. 2. Schematic for the localized electrodeposition method and images of the process (top). SEM images for the process (bottom). Images on the left display a FIB milled hole on the edge of an AFM tip covered by an $\mathrm{Al} / \mathrm{Ag}$ bilayer. On the right there are the SEM images of electrodeposited CoPt nanoparticules inside the hole previously milled. The milled hole has a size of $500 \mathrm{~nm}$ and the diameter of the deposited nanoparticle is $200 \mathrm{~nm}$.

the tip mass and resonance, or even deforms it. Therefore, large area electrodeposition by itself cannot compete with sputtering for the preparation of magnetic tips.

Our idea is then to use a local electrodeposition method rather than covering the whole cantilever with an electrodeposited film. Since the deposition would then be restricted to a small area, we could grow relatively large amounts of magnetic materials without changing significantly the total mass of the cantilever and thereby maintain its resonance frequency and quality factor. To avoid an excess of material, the tip has to be connected to a bigger electrode that will act as main deposition surface (Fig. 1). This allows us to obtain high aspect ratios via FIB sharpening while maintaining large magnetic interaction volumes, being the main problem to limit the electrodeposition to a precise region of the tip. The process we propose to solve it consists of four steps.

1) First, we deposit a $100 \mathrm{~nm} \mathrm{Al} / \mathrm{Ag}$ bi-layer on top of the cantilever via sputtering or thermal evaporation. The aluminum layer is afterwards thermally oxidized/passivated, giving a conducting layer on contact with the tip and an insulating layer on top (Fig. 2). Although this step is quite straightforward, if the aluminum layer is too thin or if it is

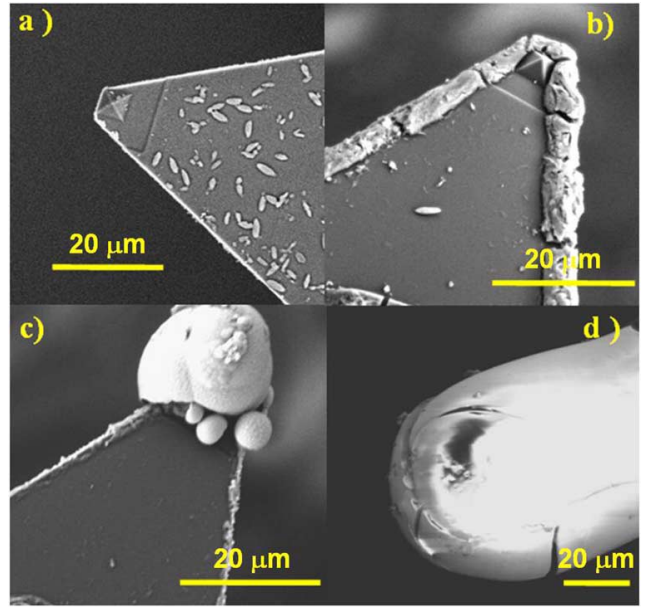

Fig. 3. Some of the problems encountered while testing electrodeposition on contact and tapping tips. (a) Deficiently oxidized aluminum layer. (b) A FIB milled hole too narrow eventually lead to high deposition at the cantilever edges. (c) Excessive deposition time. (d) Deposition of a metallic film covering the whole cantilever. This was probably due to a too low deposition $\mathrm{pH}$ (about 2) that has corroded the $\mathrm{AlO}_{\mathrm{x}}$ layer.

deficiently oxidized, there will be an additional growth of pillars and flakes all over the tip [Fig. 3(a)].

2) We use the focused ion beam to open a hole of several $100 \mathrm{~nm}$. A hole milled in an MFM tip has previously been used to increase the probe resolution [15] but in our case the location of this hole will define the localized electrodeposition region (top of the pyramidal tip in our case). If the milled hole is too small the electrodeposition will occur mostly at the edges of the tip, where the silver layer is not fully covered by the insulating $\mathrm{AlO}_{\mathrm{x}}$ layer and the electric gradient is high [Fig. 3(b)]. However, if the milled hole is big enough for the electrodeposition to grow into, large amounts of materials can be deposited at the top of the tip without having much undesired deposition over the rest of the cantilever [Fig. 3(c)].

3) The third step, the electrodeposition, is the most critical. A working electrode is prepared by making an electric contact between the tip and a copper substrate of standard size $\left(1 \mathrm{~cm}^{2}\right.$ in our case). The back of the $\mathrm{Cu}$ substrate is contacted to a wire and then varnished with an insulator, leaving only the tip side for electrodeposition (Fig. 1). We used a Pt wire as a reference electrode and the deposition was carried out potentiostatically. In potentiostatic deposition, the ionic reduction to metal will occur only if the applied potential is smaller than the reduction potential, which depends on solution concentration. The only metallic area of the tip in contact with solution is the $\mathrm{Ag}$ exposed by the hole milled through the Al/Al-oxide using the FIB. The use of relatively large working electrode areas leads to roughly reproducible deposition and deposition times while avoiding excessive current densities over the tip. Although the tips did not usually break when introduced manually into the solution, sudden movements and stirring of the solution must be avoided.

It must also be pointed out that by varying the electrodeposition parameters it is possible to roughly modify the 


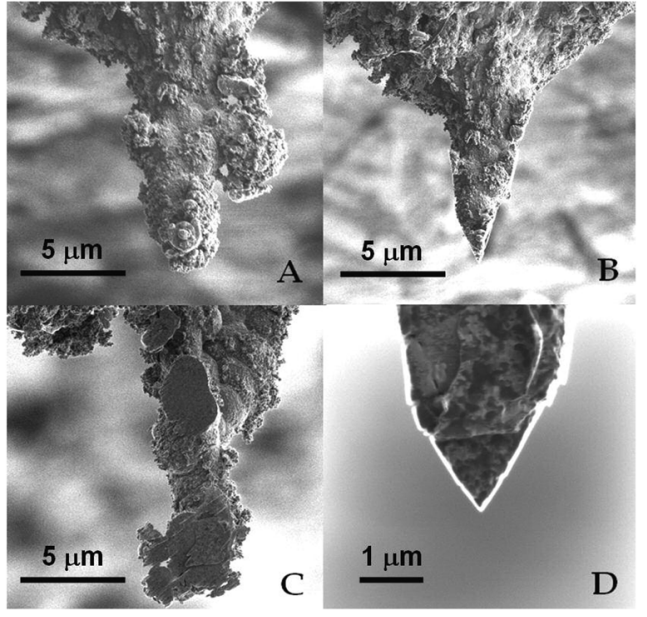

Fig. 4. Sharpening of an elongated nickel tip electrodeposited under low concentration-high voltage conditions (20 mM Ni sulphate, $8 \mathrm{~V}$, and $\mathrm{pH} 4)$. (a)-(d) Sharpening is done in two different planes at $90^{\circ}$.

geometry of the deposited tip. Electrodeposited materials from highly concentrated solutions at low $\mathrm{pHs}$ give dense smooth deposits. Raising the overpotential generally also results in smaller grain size [16]. However, high overpotentials increase the chances of cantilever break due to electrostatic effects (this possibility may be reduced by using magnetic fields during the electrodeposition) and low $\mathrm{pHs}$ $(<3)$ may lead to damage of the $\mathrm{AlO}_{\mathrm{x}}$ layer resulting on a smooth film deposition over the whole tip [Fig. 3(d)]. On the other hand, to obtain elongated, thread-like structures with high aspect ratio [17], optimal parameters are a low ionic concentration and high overpotential (Fig. 4).

In our work we have deposited both high coercivity alloys (CoPtP) and $3 \mathrm{~d}$ metals (Ni). For the electrodeposition of nickel we use a solution of $10-50 \mathrm{mM}$ of nickel sulphate with saccharin, a $\mathrm{pH}$ adjusted to 4 with boric acid and high voltages up to $8 \mathrm{~V}$. CoPtP was electrodeposited from an electrolyte containing $0.1 \mathrm{M}$ of cobalt sulfamate, $15-35 \mathrm{mM}$ of dinitrodiamimine platinum salt, $0.1 \mathrm{M}$ of diammonium hydrogen citrate and $0.1 \mathrm{M}$ of $\mathrm{NaH}_{2} \mathrm{PO}_{2}$. A $\mathrm{Ni}$ or Co foil was used as counter electrode. Plating was carried out without any agitation, although magnetic fields were used to focus the currents and avoid turbulences or precipitation at high voltages. Plating current densities of $16-40 \mathrm{~mA} / \mathrm{cm}^{2}$ were used.

4) Finally, further patterning of the deposited material and increased aspect ratio is possible by using a FIB as a sharpening tool (Fig. 4) [18]. Currents of the order of $100 \mathrm{pA}$ are enough to remove the excessive material on reasonable time scales (minutes per cut) and low Ga implantation. Furthermore, we find that sharper tips are obtained by taking away the material with lines cut across the tip, chiseling the material away, rather than milling the whole area.

When the electrodeposition covers a significant area of the cantilever, there is a displacement of up to $10 \%$ in the tapping resonant frequency due to increased cantilever mass and/or deformation leading to spring constant changes. This happens in the more heavily deposited tips, where

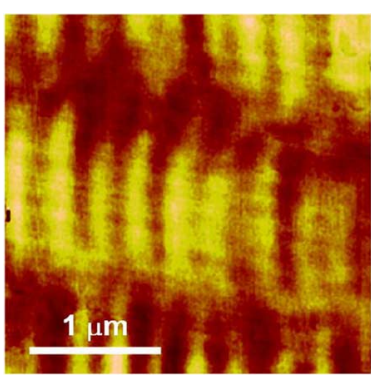

(a)

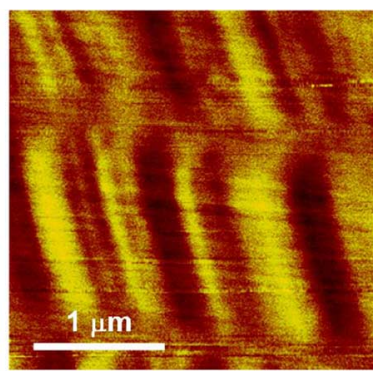

(b)
Fig. 5. (a) MFM images of a $20 \mathrm{~Gb}$ hard disk taken with an electrodeposited $\mathrm{Ni}$ tip before and (b) after FIB sharpening. Z-scale is $3^{\circ}$ (phase mode).

the deposited material has a mass comparable to that of the cantilever itself.

Since the sensitivity of a MFM tip remains constant for thicknesses above roughly $50 \mathrm{~nm}$ [19], we must emphasize that our purpose is not to make thicker magnetic films to gain in resolution, but to have a high enough amount of magnetic material even after the FIB sharpening. Furthermore given that the amount of deposited magnetic material at the tip exceeds $100 \mu \mathrm{m}^{3}$ we have enough initial material to chisel the tip into any desired shape without losing its magnetic properties.

\section{IMAGING}

All our scans have been obtained at room temperature and open atmosphere with a commercial instrument (Digital Instruments MultiMode III). As could be expected from experiments with FIB-sharpened tips, our tips give good topographic images, comparable with commercial tips on smooth samples, and even better than commercial tips in samples with a rough surface or deep features such as alumina membranes. This allows us to record images with very small lift offsets, down to $10 \mathrm{~nm}$ in smooth thin films.

The MFM images are phase mode measurements obtained with lift heights of order 50-100 nm and amplitudes of $20-70 \mathrm{~nm}$ on open air with no vibration isolation. As standard samples we chose to scan high density, in-plane recording media with a top amorphous carbon layer ( $20 \mathrm{~Gb}$ hard disk). These samples are a good sensitivity test because they have weak stray field gradients, and therefore are more difficult to image than media with perpendicular magnetization.

The magnetic resolution previous to FIB milling is not very good, particularly with soft nickel tips, where the image features are of order $100 \mathrm{~nm}$ (Fig. 5), but the signal is strong. After FIB milling the resolution is greatly enhanced.

For high coercivity CoPtP tips and in measurements taken from the same hard disks, we obtained good images over large areas, with the signal strength about a factor 3 above that of commercial tips [Fig. 6 (top)]. The tips showed even better performance on samples with relatively small magnetic moment and rough surfaces, such as when observing the stray field from single grains of diluted semiconductors [20] [average roughness of 20-30 nm and magnetic moment of order $10^{-8} \mathrm{Am}^{2}$; see Fig. 6 (bottom)], where the stray field can be clearly seen around the $200 \mathrm{~nm}$ size grains. 


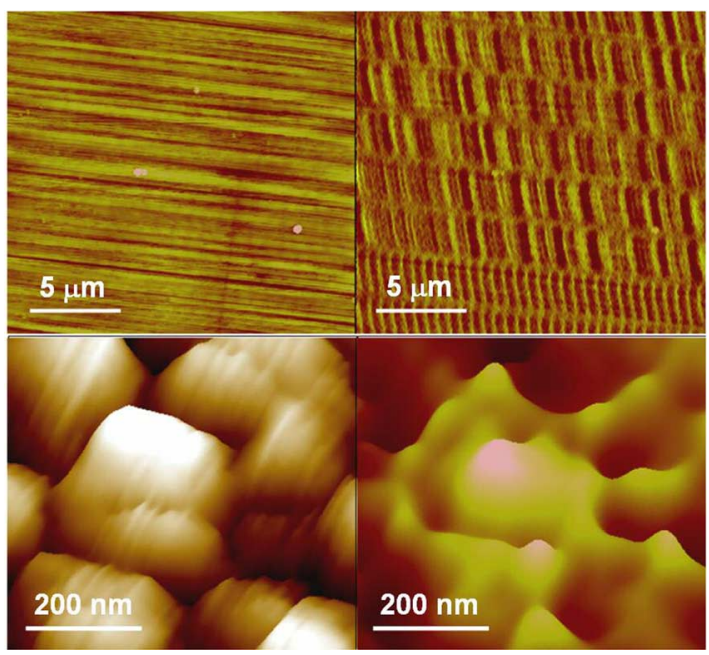

Fig. 6. AFM (top left) and MFM (top right) images of a commercial $20 \mathrm{~Gb}$ hard disk obtained with an electrodeposited and FIB sharpened CoPt tip. Z-scales are $10 \mathrm{~nm}$ and $3^{\circ}$ (phase mode). Lines on topographic image are due to polishing. AFM (bottom left) and MFM (bottom right) 3-D perspective images of an small area of a Fe-doped $\mathrm{SnO}_{2}$ film. Diagonal lines on the AFM image are an artifact due to laser interference. Background due to topographic noise has been subtracted in the MFM. Z-scales are $40 \mathrm{~nm}$ and $5^{\circ}$.

\section{CONCLUSION}

Our method combining focused ion beam milling with electrodeposition allows for the fabrication of high coercivity and aspect-ratio magnetic force microscopy tips containing a high net magnetic volume without affecting significantly the resonance of the cantilever. These tips provide high quality topographic and magnetic images at RT and open air, with applications also in the quantitative measurement of ferromagnetic resonance and magnetic nano-writing.

\section{ACKNOWLEDGMENT}

The authors would like to thank Dr. C. Fitzgerald for the PLD Fe-doped SnO film. This work was supported by Science Foundation Ireland as part of the CINSE and MANSE projects.

\section{REFERENCES}

[1] J. Lohau, S. Kirsch, A. Carl, G. Dumpich, and E. F. Wassemann, "Quantitative determination of effective dipole and monopole moments of magnetic force microscopy tips," J. App. Phys., vol. 86, p. 3410, 1999.

[2] Y. Rheem, H. Saito, and S. Ishio, "Fabrication of FePt/FeCo/FePt exchange-spring trilayer with very thin $\mathrm{FeCo}$ layer for high-resolution MFM tips," IEEE Trans. Magn., vol. 41, p. 3793, 2005.
[3] S. H. Liou and Y. D. Yao, "Development of high coercivity magnetic force microscopy tips," J. Magn. Magn. Mater., vol. 190, p. 130, 1998.

[4] A. Winkler, T. Mühl, S. Menzel, R. Kozhuharova-Koseva, S. Hampel, A. Leonhardt, and B. Büchner, "Magnetic force microscopy sensors using iron-filled carbon nanotubes," J. Appl. Phys., vol. 99, p. 104905, 2006.

[5] G. Yang et al., "Magnetic nanowire based high resolution magnetic force miscroscope probes," Appl. Phys. Lett., vol. 87, p. 123507, 2005.

[6] M. R. Koblischka, B. Hewener, U. Hartmann, A. Wienss, B. Christoffer, and G. Persh-Schuy, "Magnetic force microscopy applied in magnetic data storage technology," Appl. Phys. A, vol. 76, p. 879, 2003.

[7] L. Gao, L. P. Yue, T. Yokota, R. Skomski, S. H. Liou, H. Takahoshi, H. Saito, and S. Ishio, "Focused ion beam milled CoPt magnetic force microscopy tips for high resolution domain images," IEEE Trans. Magn., vol. 40, p. 2194, 2004.

[8] Z. Zhang, P. C. Hammel, and P. E. Wigen, "Observation of ferromagnetic resonance in a microscopic sample using magnetic resonance force microscopy," Appl. Phys. Lett., vol. 68, p. 2005, 1996.

[9] V. V. Naletov, V. Charbois, O. Klein, and C. Fermon, "Quantitative measurement of the ferromagnetic resonance signal by force detection," App. Phys. Lett., vol. 83, p. 3132, 2003.

[10] T. Onoue, M. H. Siekman, L. Abelmann, and J. C. Lodder, "Probe recording on $\mathrm{CoNi} / \mathrm{Pt}$ multilayered thin films by using an MFM tip," J. Magn. Magn. Mater., vol. 272-276, p. 2317, 2004.

[11] R. Garcia, R. V. Martinez, and J. Martinez, "Nano-chemistry and scanning probe nanolithographies," Chem. Soc. Rev., vol. 35, p. 29, 2006.

[12] F. M. F. Rhen, E. Backen, and J. M. D. Coey, "Thick-film permanent magnets by membrane electrodeposition," J. Appl. Phys., vol. 97, p. 113908, 2005.

[13] I. Zana and G. Zangari, "Magnetic propertied of electrodeposited Co-Pt thin films with very high perpendicular magnetic anisotropy," J. Magn. Magn. Mater., vol. 272, p. 1698, 2004.

[14] J. Abbou, C. Demaille, M. Druet, and J. Moiroux, "Fabrication of submicrometer-sized gold electrodes of controlled geometry for scanning electrochemical-atomic force microscopy," Anal. Chem., vol. 74, p. 6355,2002

[15] L. Folks, M. E. Best, P. M. Rice, B. D. Terris, D. Weller, and J. N. Chapman, "Perforated tips for high-resolution in-plane magnetic force microscopy," Appl. Phys. Lett., vol. 76, p. 909, 2000.

[16] E. Moti, M. H. Shariat, and M. E. Bahrololoom, "Influence of cathodic overpotential on grain size in nanocrystalline nickel deposition on rotating cylinder electrodes," J. Appl. Electrochem., to be published.

[17] T. R. Ní Mhíocháin and J. M. D. Coey, "Chirality of electrodeposits grown in a magnetic field," Phys. Rev. E, vol. 69, p. 061404, 2004.

[18] G. N. Phillips, M. H. Siekman, L. Abelmann, and J. C. Lodder, "High resolution magnetic force microscopy using focused ion beam modified tips," Appl. Phys. Lett., vol. 81, p. 865, 2002.

[19] K. Babcock, V. Elings, M. Dugas, and S. Loper, "Optimization of thin-film tips for magnetic force microscopy," IEEE Trans. Magn., vol. 30, p. 4503, 1994.

[20] C. B. Fitzgerald et al., "Magnetism in dilute magnetic oxide thin films based on $\mathrm{SnO}_{2}$," Phys. Rev. B, vol. 74, p. 115307, 2006.

Manuscript received March 03, 2008. Current version published December 17, 2008. Corresponding author: O. Céspedes (e-mail: cespius@yahoo.com). 